\title{
Efficiency simulations for the beam collimation system of the Japan Proton Accelerator Research Complex rapid-cycling synchrotron
}

\author{
Kazami Yamamoto \\ J-PARC Center, Japan Atomic Energy Agency (JAEA), Tokai-mura, Naka-gun, Ibaraki-ken, 319-1195 Japan \\ (Received 27 March 2007; revised manuscript received 6 March 2008; published 18 December 2008) \\ The $3 \mathrm{GeV}$ rapid-cycling synchrotron (RCS) of the Japan Proton Accelerator Research Complex \\ (J-PARC) project generates a $1 \mathrm{MW}$ proton beam. In operation of a high intensity hadron accelerator, the \\ most important issue is to minimize the uncontrolled loss. The RCS beam collimation system is designed \\ for this purpose and the performance is confirmed. In the present design, the physical aperture is 1.5 times \\ wider than the primary collimator aperture, so that the beam loss can be sufficiently localized under this \\ condition. The influence of positioning errors of the collimators is also estimated. Furthermore, the \\ dependence of the collimation efficiency on the impact parameter is investigated taking the growth \\ process of the beam halo into consideration. As a result, the beam loss mechanism changes according to \\ the impact parameter.
}

DOI: 10.1103/PhysRevSTAB.11.123501

PACS numbers: 29.27.Eg

\section{INTRODUCTION}

The Japan Proton Accelerator Research Complex (J-PARC) project is a cooperation of the Japan Atomic Energy Agency (JAEA) and the High Energy Accelerator Research Organization (KEK). The J-PARC accelerator complex consists of a $181 \mathrm{MeV}$ (first stage) or $400 \mathrm{MeV}$ (second stage) linac, a $3 \mathrm{GeV}$ rapid-cycling synchrotron (RCS) and a $50 \mathrm{GeV}$ main ring (MR) [1], located at the Tokai Research and Development Center of JAEA. In 2008, the accelerators RCS and MR are in the beam commissioning phase.

The RCS accelerates protons from $181 \mathrm{MeV}$ (or $400 \mathrm{MeV}$ in stage 2) to $3 \mathrm{GeV}$ kinetic energy at $25 \mathrm{~Hz}$ repetition rate. The average beam current is $0.333 \mathrm{~mA}$ and the design beam power is $1 \mathrm{MW}$. The RCS proton beam is extracted and either sent to the MR or to the spallation neutron target in the Material and Life Science Facility. In designing such a high intensity hadron accelerator, the most important issue is to minimize the uncontrolled loss around the accelerator. To allow hands-on maintenance of the accelerator components, the average beam loss around the accelerator should be kept below 1 Watt per meter $[2,3]$. This value corresponds to a loss of $10^{-6}$ of the $1 \mathrm{MW}$ full beam power per meter. Since it is very difficult to control the beam loss to such a low level, a beam collimation system is arranged in order to remove the beam halo and to localize the beam loss. Here the design and the validity check of the RCS beam collimation system is presented.

\section{RCS COLLIMATION SYSTEM DESIGN}

In the RCS, a two-stage collimation system [4-6] is applied for the transverse collimation. Moreover, an additional primary collimator is designed for the longitudinal collimation. The two-stage collimation system consists of a primary collimator, which scatters the halo particles, and secondary collimators, which absorb those scattered particles. By scattering with a thin primary collimator, the impact parameter at the secondary collimators can be enlarged. Therefore the chance that a halo particle will leak from the secondary collimators is reduced. Moreover, the beam loss can be localized at places where the phase advance from the primary collimator is less than 180 degrees.

The transverse primary and secondary collimators are installed in the half of the injection straight section, which has a dispersion-free optic. The transverse primary collimator consists of horizontal and vertical scatterers, and is installed in the entrance to the collimator region. The five secondary collimators are installed in the downstream of the transverse primary collimator. If particle scattering occurs only in one dimension, the ideal phase advance of the secondary collimators is expressed by the following simplified equation [7]:

$$
\mu_{1}=\arccos \frac{n_{1}}{n_{2}}, \quad \mu_{2}=\pi-\mu_{1} .
$$

Here, $n_{1}$ and $n_{2}$ are the acceptance of the primary and secondary collimators in the normalized phase space. In the RCS, since $n_{1} / n_{2}$ is $0.9\left(n_{1}{ }^{2}=324, n_{2}{ }^{2}=400\right)$, the ideal phase advance would be approximately 26 degrees and 154 degrees, respectively. However, since the particle scattering of the other dimensions of phase space is generated at an arbitrary position, the scattered particles are lost in places other than those with the above-mentioned phase relation. Thus additional secondary collimators were appended. Table I shows the phase advance between the primary and each secondary collimator at the nominal operation point.

The longitudinal collimation system is composed of a longitudinal primary collimator and the above two-stage 
TABLE I. The phase advance between the primary and secondary collimators.

\begin{tabular}{lccccc}
\hline \hline & Sec. No. 1 & Sec. No. 2 & Sec. No. 3 & Sec. No. 4 & Sec. No. 5 \\
\hline Horizontal (degree) & 14.1 & 68.5 & 88.7 & 101.6 & 110.5 \\
Vertical (degree) & 15.7 & 27.3 & 44.0 & 99.6 & 112.5 \\
\hline \hline
\end{tabular}

(transverse primary and secondary) collimator. The longitudinal collimator is placed in order to remove the longitudinal halo between injection bunches. The injected beam is cut off with the linac chopper, but some particles still pass through. These particles will become the longitudinal halo by the synchrotron oscillation in the RCS. The longitudinal primary collimator is installed in the arc section in front of the injection point. The longitudinal halo particles, which are scattered by the longitudinal primary collimator, are diffused between the aperture of the transverse collimators and the physical aperture of other components, and can pass through the vacuum pipe until they hit the transverse collimators again.

Both primary collimators aperture restrict the emittance to $324 \pi \mathrm{mm}$ mrad and $1 \%$ momentum deviation. All secondary collimator apertures are set to $400 \pi \mathrm{mm} \mathrm{mrad}$. These values are chosen to prevent the beam from reaching the secondary collimator before the halo particles hit the primary collimator. All other vacuum pipes are designed for a beam with more than $486 \pi \mathrm{mm}$ mrad emittance and $1 \%$ momentum deviation. Figure 1 shows the location of the collimation system in the RCS, and the Twiss parameters are shown in Fig. 2.

The transverse primary collimator and each of the secondary collimators are consisting of four plates-one for each direction (upper, lower, right, and left). The longitu-

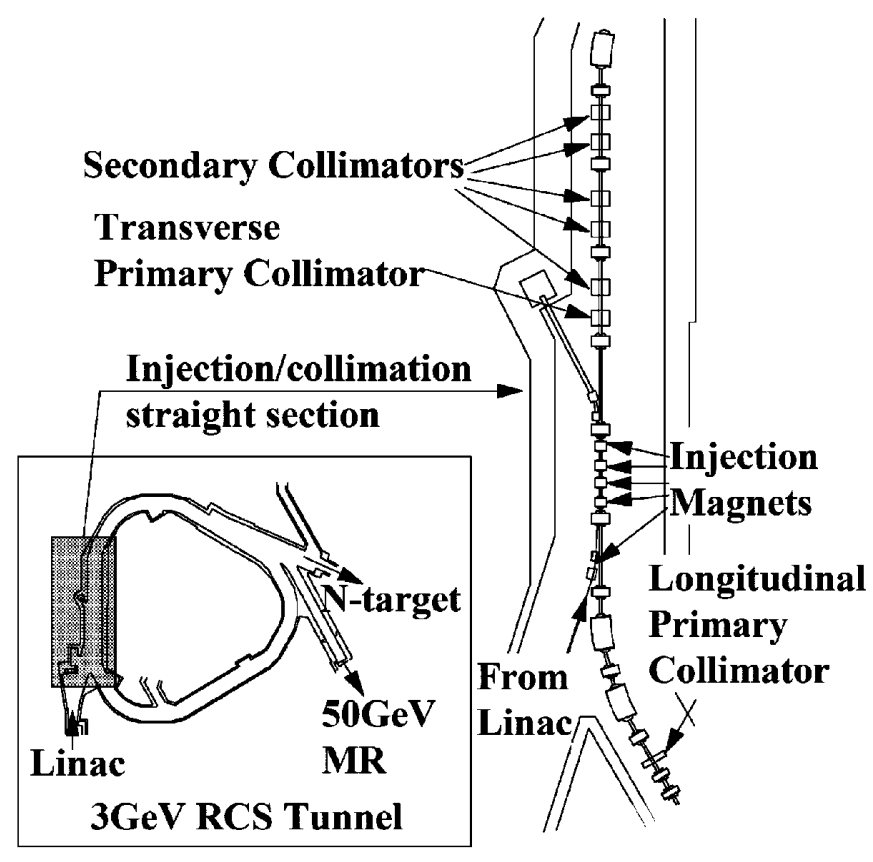

FIG. 1. The location of the collimation system in the RCS. dinal primary collimator is made from two horizontal plates (right and left). Height and width of these plates are $100 \mathrm{~mm} \times 100 \mathrm{~mm}$. The material of the transverse primary collimator is $1 \mathrm{~mm}$ thick tungsten. The longitudinal primary collimator is made from $0.1 \mathrm{~mm}$ thick graphite sheets. These materials were chosen for their availability and high melting point. The thickness was decided from the necessary particle scattering angle. According to Rossi and Greisen [8], the rms scattering angle can be expressed by a simple formula. This formula gives the rms scattering angle in terms of the radiation length $L_{R}$ and thickness $L$ of the target:

$$
\theta_{\mathrm{rms}}=\frac{E_{s}}{p c \beta} \sqrt{\frac{L}{L_{R}}} .
$$

Here $p$ and $c \beta$ are the momentum and velocity of the incident particle, respectively. $E_{s}$ is a constant whose value is $21 \mathrm{MeV}$. If the atomic number of the target material is more than 20, Eq. (2) is modified as follows [9]:

$$
\theta_{\mathrm{rms}}=\frac{E_{s}^{\prime}}{p c \beta} \sqrt{\frac{L}{L_{R}}}\left(1+0.125 \log _{10} \frac{L}{L_{R}}\right) .
$$

Then $E_{s}^{\prime}$ is revised to be $17.5 \mathrm{MeV}$. For $181 \mathrm{MeV}$ protons $\theta_{\text {rms }}$ of the transverse collimator is estimated to be $26.2 \mathrm{mrad}$, and $\theta_{\text {rms }}$ of the longitudinal primary collimator is $0.72 \mathrm{mrad}$. For $400 \mathrm{MeV}$ protons $\theta_{\text {rms }}$ of the transverse collimator is $12.81 \mathrm{mrad}$, and $\theta_{\text {rms }}$ of the longitudinal primary collimator is $0.35 \mathrm{mrad}$. The increased emittance due to the scattering is

$$
\varepsilon=\varepsilon_{0}+\beta_{\text {pri }} \theta^{2},
$$

where $\varepsilon_{0}$ and $\varepsilon$ are the emittance before and after scattering, $\beta_{\text {pri }}$ is the $\beta$ function at the primary collimator, and $\theta$ is the scattering angle. Since almost all scattered particles are in the $3 \sigma$ range, it is assumed that $\theta$ by the longitudinal primary collimator is $1.05 \mathrm{mrad}$. Then the increased emittance due to the scattering at the longitudinal primary collimator is estimated to be $346 \pi \mathrm{mm} \mathrm{mrad}$, which is smaller than the physical aperture. Thus, the scattered particles can pass through the vacuum pipe until they hit the transverse collimators again. The increased emittance due to the transverse primary collimator is very large, enough to concentrate the scattered particles in the secondary collimators.

The secondary collimator plates are made from $200 \mathrm{~mm}$ thick copper. This value was chosen so that several hundred $\mathrm{MeV}$ protons can be stopped adequately. The heat load at 

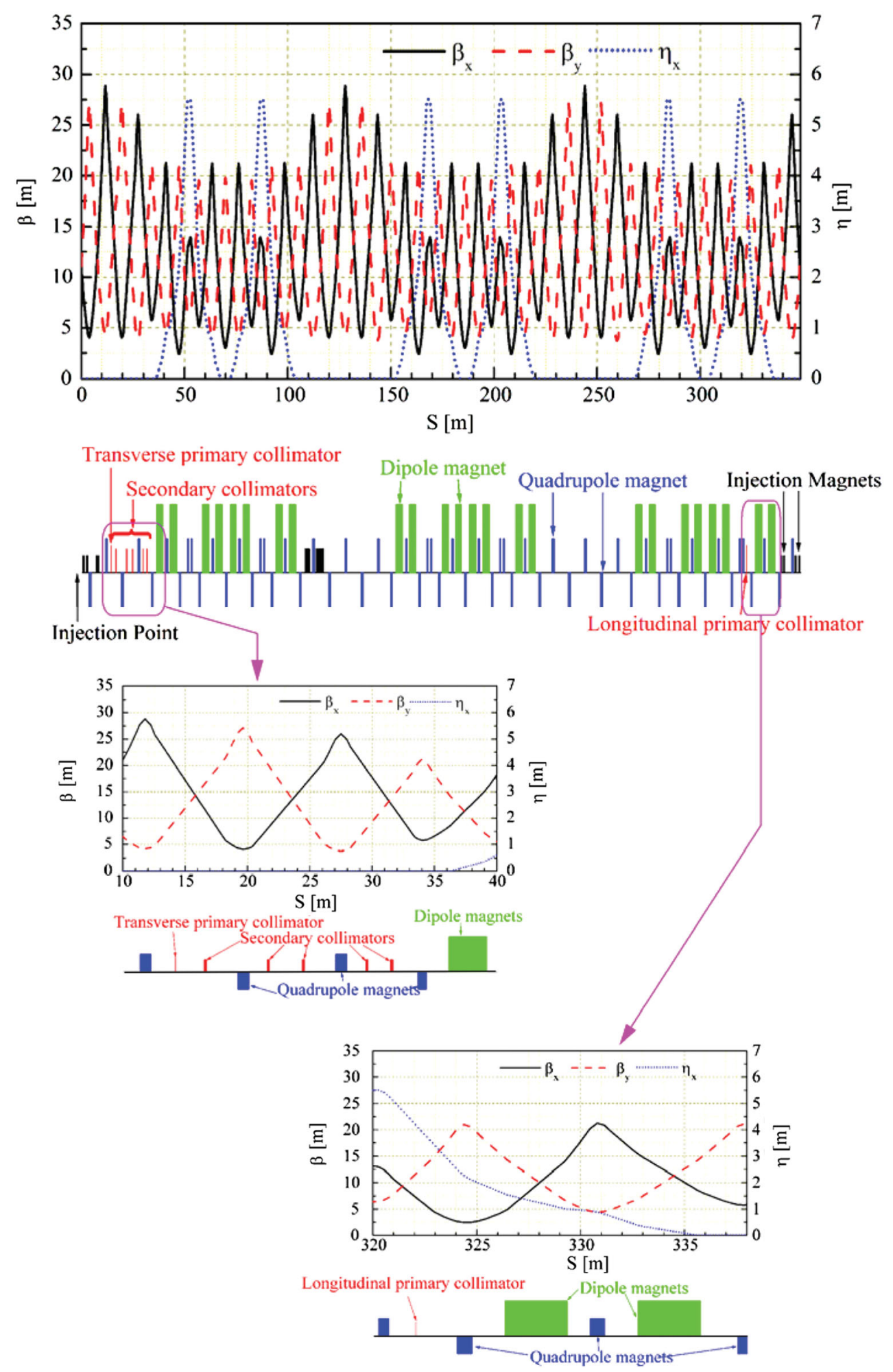

FIG. 2. (Color) RCS Twiss parameters.

one secondary collimator plate is estimated to be about $700 \mathrm{~W}$. This includes $20 \%$ margin. The material of the secondary collimator is chosen for high density and high thermal conductivity in order to release the $700 \mathrm{~W}$ heat load. All collimator plates can be moved individually.

\section{SIMULATION}

By using the above-mentioned collimation parameters, the performance of the RCS collimation system was estimated under various conditions. The evaluation was car- 
ried out by using the STRUCT code [10]. The STRUCT code was developed at Fermi National Accelerator Laboratory. In this code, linear transfer matrices model the particle transport. When a particle hits the collimator, the STRUCT code calculates the effect of the energy loss and the multiple coulomb scattering that the particle experiences. As a result, if the energy decreases below a certain value, it is assumed that the particle is lost. Furthermore, a particle is lost when it hits the vacuum chamber at a place other than the collimators, too. The coordinates and energy of the lost particles are recorded. The beam loss point and the amount of beam loss can be evaluated from this information. Moreover, this information is delivered to a Monte Carlo simulation such as MARS [11], and used for evaluation of the radiation level of the collimator, the design of its shielding [12], and the design of the cooling system $[13,14]$.

In order to use the code STRUCT, it is necessary to supply the coordinates of the halo particles as the initial condition. First of all, two halo distributions were assumed extending in the transverse and longitudinal phase space. The transverse halo particles were distributed in the range from 324 to $364 \pi \mathrm{mm}$ mrad transverse emittance and from $-1 \%$ to $1 \%$ momentum within the rf bucket shape. Longitudinal halo particles were set to be in the range from $1 \%$ to $1.5 \%$ momentum and to be distributed up to $324 \pi \mathrm{mm}$ mrad transverse emittance. Any transverse halo particle, which has large horizontal emittance, has small vertical emittance, and vice versa. This represents the anticorrelated painting process. On the other hand, a thin distribution was assumed in investigating the impact parameter dependence of the collimator in Sec. IV C.

All calculation models assume that the total beam loss is $4 \mathrm{~kW}$. In these calculations, the collimation efficiency is defined as follows:

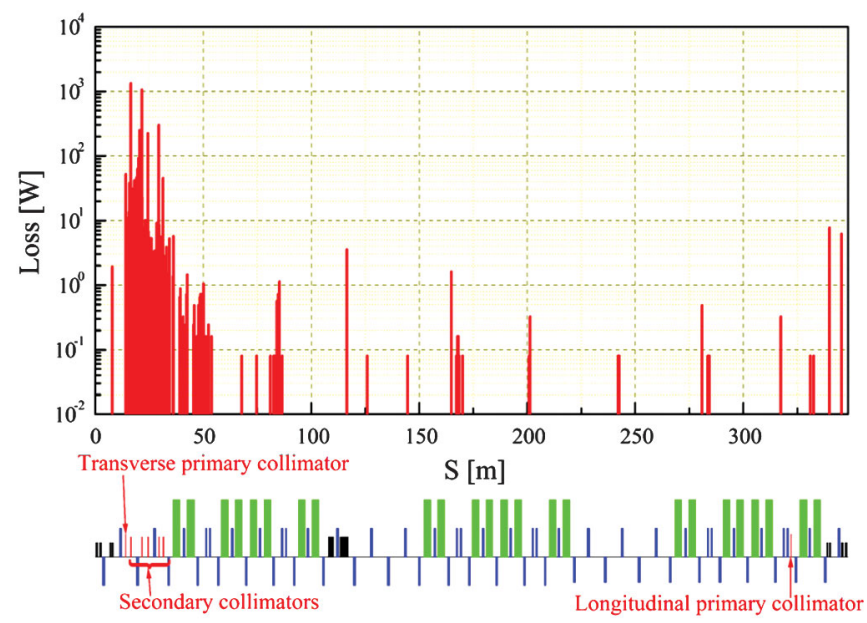

FIG. 3. (Color) Beam loss distribution along the RCS in the longitudinal direction.

$$
\begin{aligned}
& \text { collimation efficiency } \\
& =\frac{\text { beam loss }[\mathrm{W}] \text { in the collimator region }}{\text { beam loss }[\mathrm{W}] \text { in whole ring }} .
\end{aligned}
$$

This value is an indicator of collimation performance. An example of the beam loss distribution is shown in Fig. 3. The transverse halo collimation was done at the nominal operation point $\left(\nu_{x}, \nu_{y}\right)=(6.68,6.27)$ assuming no errors. As a result, the collimation efficiency is about $98 \%$. The maximum loss power at one collimator is $1.2 \mathrm{~kW}$ and the maximum heat load on one plate is $540 \mathrm{~W}$.

\section{RESULTS AND DISCUSSION}

\section{A. Ratio of physical aperture to collimator aperture}

In order to control the beam loss localization effectively, it is very important to secure a physical aperture of sufficient size relative to the collimator apertures. The collimation efficiency dependence on the ratio of the physical aperture to the collimator aperture was estimated for the two tunes $\left(\nu_{x}, \nu_{y}\right)=(6.72,6.35)$ and $(6.68,6.27)$, which were considered as RCS operation points.

In these calculations, all primary collimators were fixed at $324 \pi \mathrm{mm} \mathrm{mrad}$ and $1 \%$ momentum acceptance. All secondary collimators were fixed at $400 \pi \mathrm{mm}$ mrad. The physical apertures of other components were varied from $70 \%$ to $130 \%$ of the design value. Figure 4 shows the variations in the aperture size of the RCS, and Fig. 5 shows the collimation efficiency for various ratios of the physical to the collimator aperture.

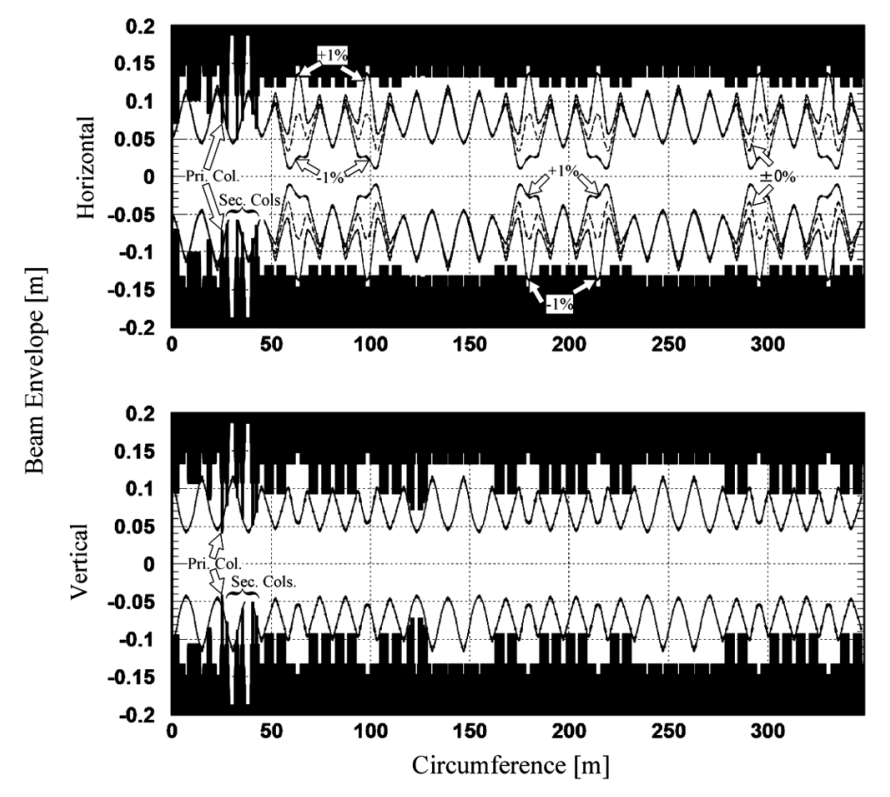

FIG. 4. The aperture design and the beam envelope around the ring. The beam envelope is assumed to be $486 \pi \mathrm{mm}$ mrad. The upper plot shows the horizontal plane including the effect of $1 \%$ momentum dispersion. The lower plot is the vertical plane. All dimensions are in meters. 


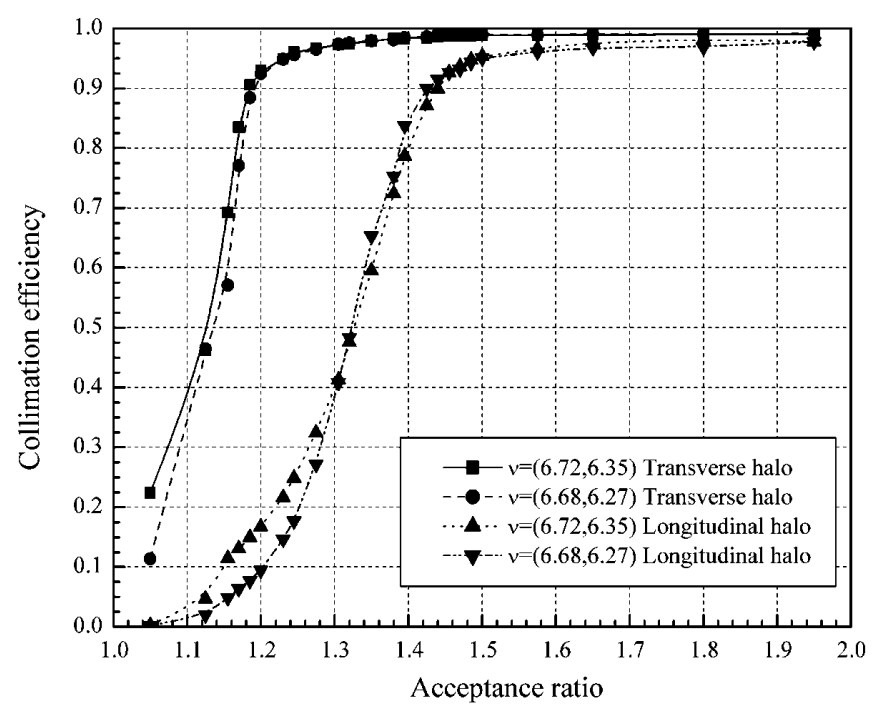

FIG. 5. The collimation efficiency dependence on the aperture ratio.

A value of $70 \%$ of the design component aperture size is almost comparable to size of the primary collimators, so in this case the collimation efficiencies of all operation points were not good. The collimation efficiency improved with wider physical aperture. However, the incremental improvement reduced to almost zero when approaching the design aperture ratio $(=1.5)$. Consequently, the reason for the saturation of the collimation efficiency was investigated. Figure 6 shows the scattered particle motion in the horizontal phase space during the collimation process. The particles scattered by the horizontal primary collimator [Fig. 6(a)] were absorbed in each secondary collimator [Figs. 6(b)-6(d)]. However, some particles leaked from the $486 \pi \mathrm{mm} \mathrm{mrad}$. acceptance range and were lost when the beam entered the arc [Figs. 6(e) and 6(f)]. This was because the number of particles suffering a large energy loss through angle scattering was small. It is not effective to increase the ratio of the physical aperture to the collimator aperture to a value higher than the present design value. From the viewpoint of collimation efficiency, the design aperture is appropriate.

\section{B. Effect of errors}

Next the effect of errors was considered. An error of the primary collimator position will substantially change the collimator acceptance. For example, if there is a $1 \mathrm{~mm}$ error in position, the primary collimator acceptance will change by $4 \%$. If the primary collimator sticks out by $1 \mathrm{~mm}, 4 \%$ of the beam emittance will be deleted. If the primary collimator is pulled back $1 \mathrm{~mm}$, the ratio of the physical aperture to the collimator aperture will become $4 \%$ smaller. From the results of 4.1, the estimated efficiency of the longitudinal collimation becomes $3.5 \%$ worse with $1 \mathrm{~mm}$ position error.
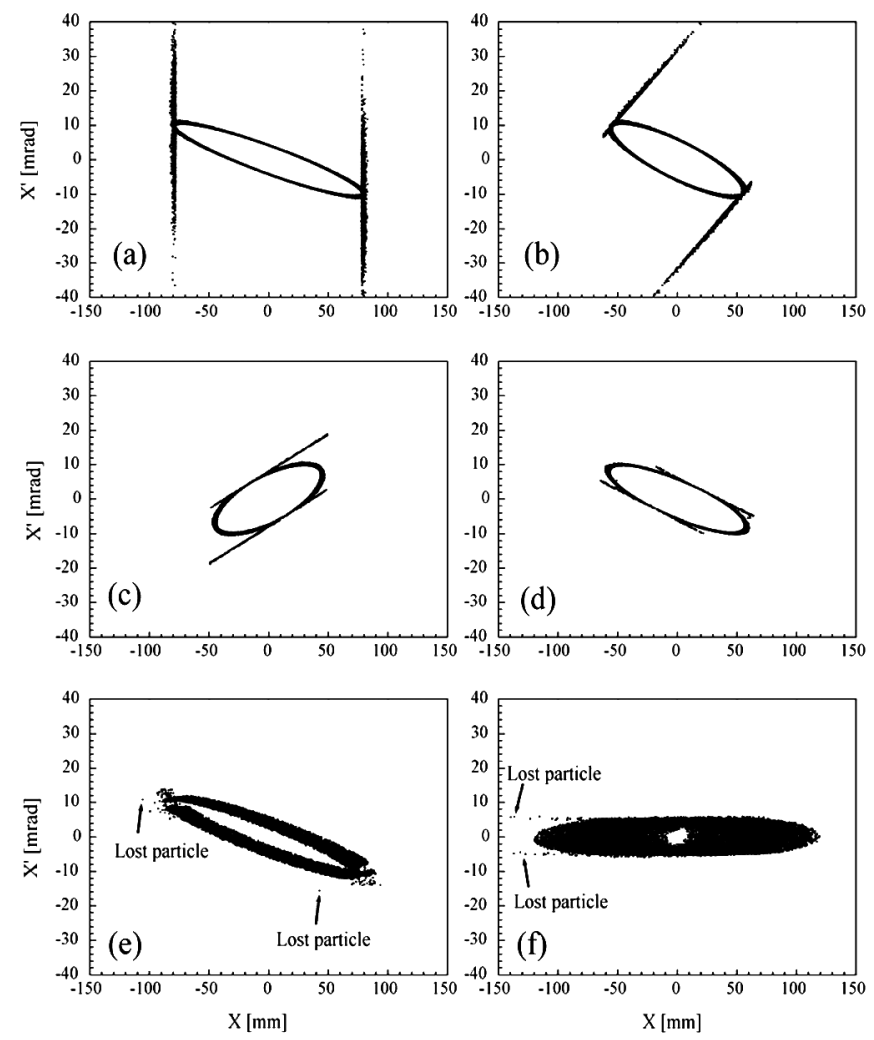

FIG. 6. Scattered particle motion in the horizontal phase space. Here a horizontal halo was assumed. Plots at the following locations are shown: (a) at the transverse primary collimator; (b) at the first secondary collimator; (c) at the third secondary; (d) at the last (5th) secondary collimator; (e) at the quadrupole magnet after two dipole magnets; (f) at the maximum dispersion point of the first arc after the collimators.

The influence of an error of the secondary collimator position is estimated considering the following two points. (i) If the secondary collimators stick out, halo particles may hit the secondary collimators before being scattered by the primary collimator. (ii) It becomes easy for scattered particles to escape if the secondary collimators are pulled back.

Both cases decrease the collimation efficiency. Subsequently, the influence of the positional and rotational errors of the secondary collimators on the efficiency was investigated. The positional and rotational errors were assumed to be the same direction and distance for all secondary collimators. The degree of closed orbit distortion (COD) due to shift of the central axis of the quadrupole magnets was also taken into account. The COD was assumed to be $3 \mathrm{~mm}$ as a reasonable worst case. The results of the evaluation for transverse collimation are shown in Fig. 7.

One result is that, even with a $1 \mathrm{~mm}$ positioning error, the collimation efficiency does not decrease. The collimation efficiency slightly decreases about $0.3 \%$ with a $3 \mathrm{~mm}$ positioning error. A $5 \mathrm{~mm}$ positioning error causes an 


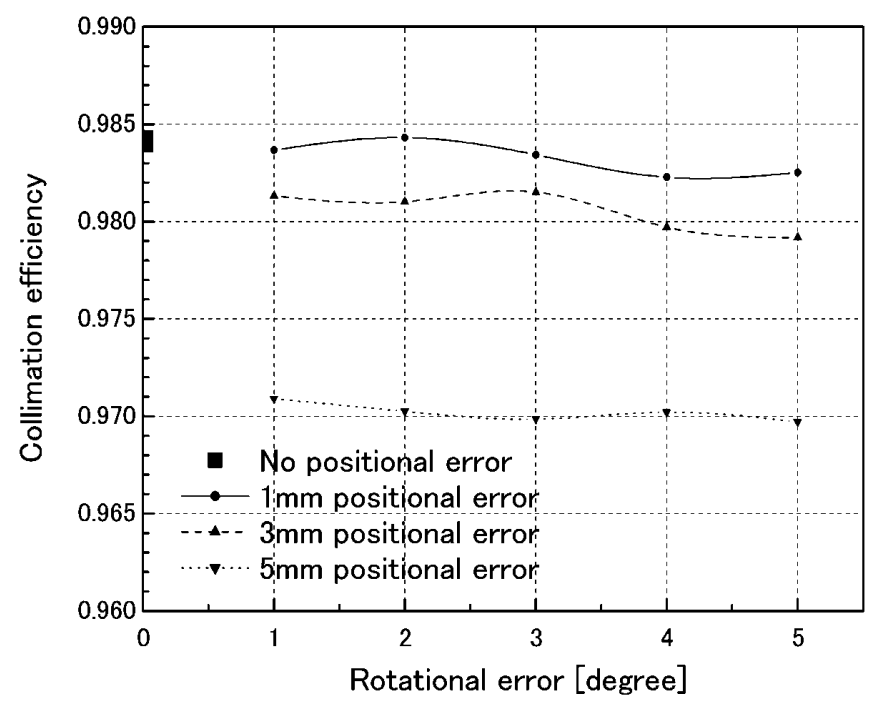

FIG. 7. The influence of errors on collimation efficiency.

efficiency decrease of more than $1 \%$. On the other hand, a rotational error of less than 5 degrees shows little influence.

\section{Impact parameter}

The actual halo distribution strongly depends on the emittance growth rate. If the growth rate is low, the impact parameter of the halo particle at the primary collimator is small. If the growth rate is high, the impact parameter becomes large. In order to estimate the influence of the growth rate, the impact parameter was changed. Figure 8 shows the collimation efficiency dependence on the impact parameter. The collimation efficiency decreases with increasing impact parameter in the range from 0.3 to $30 \mu \mathrm{m}$.

To investigate the reason for this tendency, the angular distribution and the momentum loss of the scattered particles were analyzed, and the turn number, at which particles that had been scattered first at primary collimators were

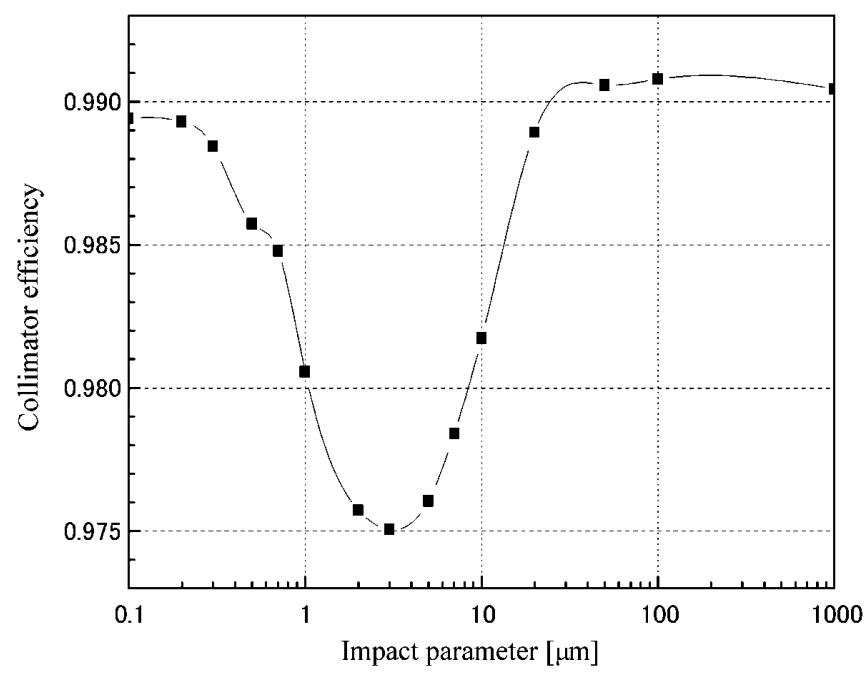

FIG. 8. The influence of the impact parameter on efficiency.

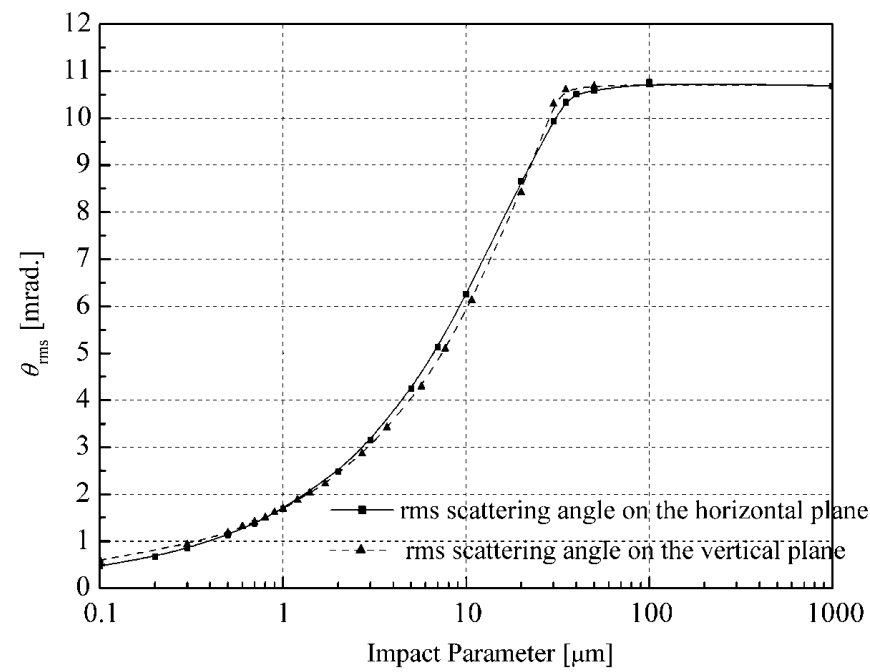

FIG. 9. The rms scattering angle dependence on impact parameter.

lost, was recorded. Figure 9 shows the rms scattering angle of a projectile on the horizontal and vertical plane. If the impact parameter is small, the rms scattering angle also is small, because many particles do not pass completely through the primary collimator and escape from it. The rms scattering angle becomes large with increased impact parameter. The angle increases quite rapidly if the impact parameter exceeds $1 \mu \mathrm{m}$, and levels off at $30 \mu \mathrm{m}$. Figure 10 shows the average momentum loss at the primary collimator. The average momentum loss has the same shape as that of the rms scattering angle.

The average turn number, at which halo particles that had been scattered first at the primary collimators were lost, is shown in Fig. 11. For small impact parameter, the average turn number increases with increasing impact

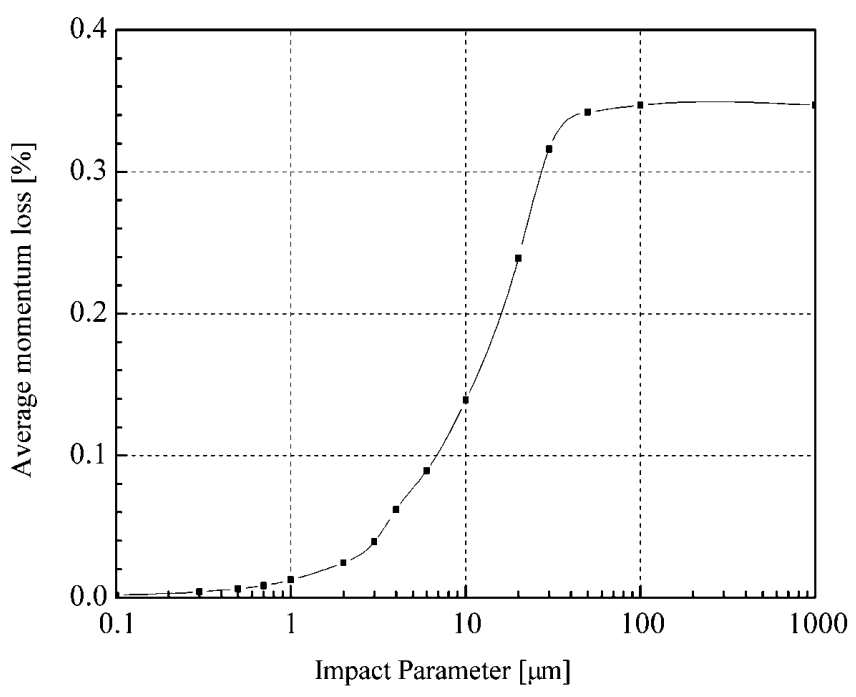

FIG. 10. The average momentum loss dependence on impact parameter. 


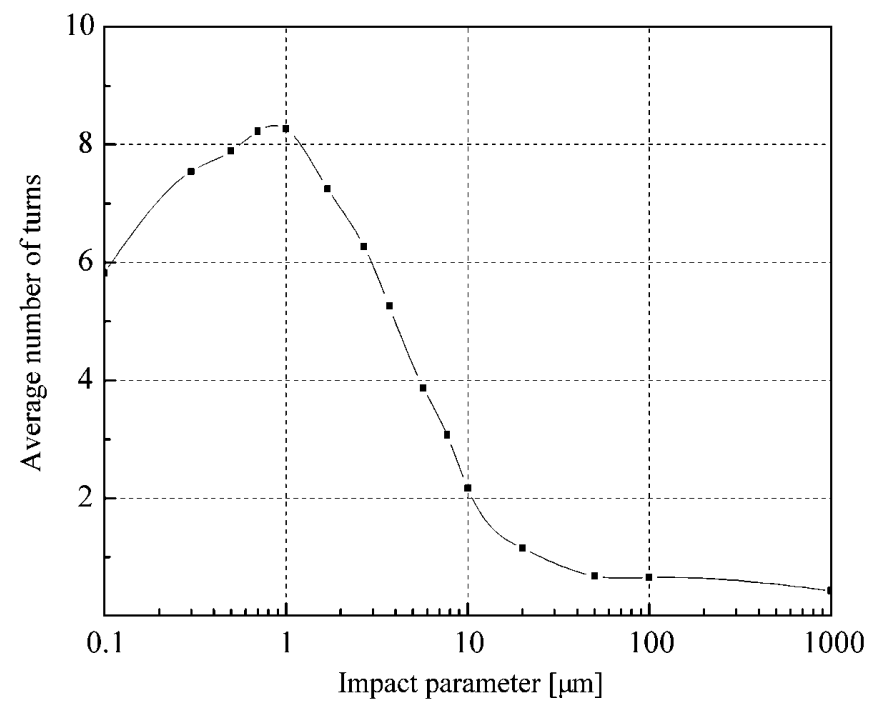

FIG. 11. The average turn number at which the particle is lost after scattering.

parameter. The peak is at $1 \mu \mathrm{m}$. After that peak, the average turn number decreases and levels off at about $30 \mu \mathrm{m}$.

Judging from these results, it is likely that there are three different beam loss mechanisms according to the impact parameter: (i) If the impact parameter is less than $1 \mu \mathrm{m}$, the scattering angle and energy loss are very small. Therefore the scattered particle can continue circulating until it hits the collimator again. The second or later hit happens with much larger impact parameter, then the large scattering angle results in a high capture efficiency. If the impact parameter is between 1 and $30 \mu \mathrm{m}$, the turn number at which the particle is lost after scattering decreases with increasing scattering angle, but the scattered particle can still pass several turns. Analyzing the trajectory of these particles shows that the transverse emittance after scattering by the primary collimator is about $500 \pi \mathrm{mm}$ mrad. Therefore, these particles are lost by the extraction kickers and injection bump magnets. These components are designed for $486 \pi \mathrm{mm}$ mrad. acceptance and this is the narrowest acceptance after the collimator. The minimum value of the efficiency appears due to these particles at the acceptance limit. If the impact parameter is more than $30 \mu \mathrm{m}$, the average turn number at which the particle is lost becomes less than 2. In other words, almost all particles are absorbed at the secondary collimators just after scattering. The efficiency improves again.

\section{CONCLUSIONS}

The performance of the design of the beam collimation system of J-PARC RCS is confirmed under various con- ditions. The beam loss can be sufficiently localized with the present aperture design. A $1 \mathrm{~mm}$ positioning error of the primary collimators causes a decrease of collimation efficiency. On the other hand, a $1 \mathrm{~mm}$ positioning error of the secondary collimators does not affect the collimation efficiency. Furthermore, the efficiency dependence on the impact parameter was investigated, taking the halo growth process into consideration. It appears that the beam loss mechanism differs according to the impact parameter. From these studies, the RCS collimator mechanism was designed to localize the beam loss with enough precision. The upper and lower side (and right and left side) collimators are adjustable independently. If the beam control is insufficient, the collimator aperture can be narrowed, which increases efficiency.

\section{ACKNOWLEDGMENTS}

I am very grateful to Dr. Alexander Drozhdin to offer his code and help. I also want to thank Professor K. Satoh and Professor Y. Irie for their important advice.

[1] JAERI-Tech Report No. 2003-34, edited by Y. Yamazaki, 2003; KEK Report No. 2002-13, 2002.

[2] "Beam Halo and Scraping" edited by N. V. Mokhov and W. Chou, in the 7th ICFA Mini-workshop on High Intensity High Brightness Hadron Beams, Lake Como, Wisconsin, 1999.

[3] JHP Project Office, KEK Report No. 97-16, JHF-97-10, 1998.

[4] T. Trenkler and J. B. Jeanneret, Part. Accel. 50, 287 (1995).

[5] J. B. Jeanneret, Phys. Rev. ST Accel. Beams 1, 081001 (1998).

[6] P. J. Bryant, CERN Report No. 94-01, 1994.

[7] M. Seidel, DESY Report No. 94-103, 1994.

[8] B. Rossi and K. Greisen, Rev. Mod. Phys. 13, 240 (1941).

[9] V. L. Highland, Nucl. Instrum. Methods 129, 497 (1975).

[10] A. Drozhdin and N. V. Mokhov, Report No. SSCL-MAN0034, 1994.

[11] N. V. Mokhov, Report No. Fermilab-FN-628, 1995.

[12] N. Nakao, N. V. Mokhov, K. Yamamoto, Y. Irie, and A. Drozhdin, Radiation Protection Dosimetry 116, 85 (2005).

[13] K. Yamamoto and M. Kinsho, in Proceedings of the 21st Particle Accelerator Conference, Knoxville, 2005 (IEEE, Piscataway, NJ, 2005), pp. 1365-1367.

[14] K. Yamamoto, M. Okazaki, Y. Hirooka, Y. Takeuchi, A. Nakamura, H. Hanaue, and M. Abe, in Proceedings of the 10th European Particle Accelerator Conference, Edinburgh, Scotland, 2006 (EPS-AG, Edinburgh, Scotland, 2006), pp. 3200-3202. 